\section{Testergebnis, falsch-positives}

R.-D. Hilgers ${ }^{1}$, N. Heussen ${ }^{1}$ und S. Stanzel ${ }^{2}$

${ }^{1}$ Institut für Medizinische Statistik, Universitätsklinikum der RWTH Aachen, Aachen, Deutschland

${ }^{2}$ Heidelberg, Deutschland

Synonym(e) Falsch-positiver Test

Englischer Begriff false-positive test; false-positive test result

Definition Ein falsch-positives Testergebnis eines diagnostischen Tests liegt vor, wenn tatsächlich Gesunde ein positives Testergebnis aufweisen.
Beschreibung Ein falsch-positives Testergebnis stellt eine der beiden Fehlentscheidungen des diagnostischen Tests ( $\triangleright$ Test, diagnostischer) dar. In Analogie zum statistischen Test ( $\triangleright$ Test, statistischer) bezeichnet man zuweilen ein falsch-positives Testergebnis auch als einen $\triangleright$ Fehler 1 . Art. Anhand der Rate der falsch-positiven Testergebnisse lässt sich die Genauigkeit ( $\triangleright$ Accuracy, diagnostische) des Tests bewerten. Die Anzahl falsch-positiver Testergebnisse wird üblicherweise durch den Wert b bestimmt (Bezeichnungen s. Tabelle im Stichwort $>$ Vierfeldertafel).

\title{
Literatur
}

Hilgers R-D, Bauer P, Scheiber V (2002) Einführung in die Medizinische Statistik. Springer, Berlin/Heidelberg/New York 